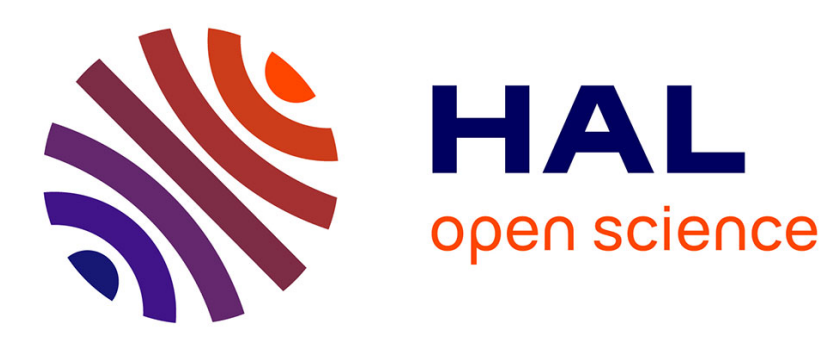

\title{
Visibly Pushdown Transducers with Look-Ahead
}

Emmanuel Filiot, Frédéric Servais

\section{To cite this version:}

Emmanuel Filiot, Frédéric Servais. Visibly Pushdown Transducers with Look-Ahead. [Research Report] 2011. inria-00573965v2

\section{HAL Id: inria-00573965 https://hal.inria.fr/inria-00573965v2}

Submitted on 25 Apr 2011

HAL is a multi-disciplinary open access archive for the deposit and dissemination of scientific research documents, whether they are published or not. The documents may come from teaching and research institutions in France or abroad, or from public or private research centers.
L'archive ouverte pluridisciplinaire HAL, est destinée au dépôt et à la diffusion de documents scientifiques de niveau recherche, publiés ou non, émanant des établissements d'enseignement et de recherche français ou étrangers, des laboratoires publics ou privés. 


\title{
Visibly Pushdown Transducers with Look-Ahead
}

\author{
Emmanuel Filiot $^{1} \quad$ Frédéric Servais $^{1}$ \\ ${ }^{1}$ Université Libre de Bruxelles
}

\begin{abstract}
Visibly Pushdown Transducers (VPT) form a subclass of pushdown transducers. In this paper, we investigate the extension of VPT with visibly pushdown look-ahead $\left(\mathrm{VPT}_{\mathrm{la}}\right)$. Their transitions are guarded by visibly pushdown automata that can check whether the well-nested subword starting at the current position belongs to the language they define. First, we show that $\mathrm{VPT}_{\mathrm{la}}$ are not more expressive than VPT, but are exponentially more succinct. Second, we show that the class of deterministic VPT $\mathrm{V}_{\mathrm{la}}$ corresponds exactly to the class of functional VPT, yielding a simple characterization of functional VPT. Finally, we show that while $\mathrm{VPT}_{\text {la }}$ are exponentially more succinct than VPT, checking equivalence of functional VPT $\mathrm{V}_{\mathrm{a}}$ is, as for VPT, EXPTIME-C. As a consequence of these results, we show that for any functional VPT there is an equivalent unambiguous one.
\end{abstract}

\section{Introduction}

Visibly pushdown transducers (VPT) [12,8] form an interesting subclass of pushdown transducers (PT). Several problems that are undecidable for PT are decidable for VPT, noticeably: functionality is decidable in PTIME, $k$-valuedness in NPTIME and equivalence of functional VPT is EXPTIME-C [8].

Visibly pushdown machines [1], automata or transducers, are pushdown machines such that the behavior of the stack, i.e. whether it pushes or pops, is visible in the input word. Technically, the input alphabet is partitioned into call, return and internal symbols. When reading a call the machine must push a symbol on the stack, when reading a return symbol it must pop and when reading an internal symbol it cannot touch the stack. The partitioning of the input alphabet induces a nesting structure of the input words [2]. A call symbol delimits an additional level of nesting, while a return symbol is a position in the word that ends a level of nesting. A word is well-nested if each call, respectively each return, has a matching return, respectively a matching call. Visibly pushdown transductions are transductions can be defined by VPT ${ }^{1}$.

In this paper, one of our motivations is to give a simple characterization of functional VPT that can be checked easily. Deterministic VPT are not expressive enough to capture all functional VPT, as for instance swapping the first and last letters of a word cannot be done deterministically. Instead of non-determinism, we show that some limited inspection of the longest well-nested subword starting at the current position (called the current well-nested prefix) is required to capture (non-deterministic) functional VPT. More precisely, we show that functional VPT-transductions are captured by deterministic VPT extended with visibly pushdown look-aheads that inspect the current well-nested prefix. Moreover, inspecting the current well-nested prefix is somehow the minimal necessary information to capture all functional VPT.

\footnotetext{
${ }^{1}$ In this paper when it is clear from the context, we also use VPT to denote visibly pushdown transductions
} 
In this paper, we therefore introduce and investigate the class of VPT with visibly pushdown look-ahead. A VPT with visibly pushdown look-ahead $\left(\mathrm{VPT}_{\mathrm{la}}\right)$ is a VPT such that call transitions are guarded with visibly pushdown automata (VPA). When reading a call at position $i$, a $\mathrm{VPT}_{\mathrm{la}}$ can apply a call transition provided the longest well-nested word starting at position $i$ is included in the language of the VPA of the transition. Our main contributions are the following:

1. $\mathrm{VPT}_{\mathrm{la}}$ are as expressive as $\mathrm{VPT}$, but exponentially more succinct.

For this we present an exponential construction that shows how a VPT can simulate look-aheads. Moreover we show this exponential blow-up is unavoidable.

2. Deterministic $\mathrm{VPT}_{\mathrm{la}}$ and functional $\mathrm{VPT}$ are equally expressive.

This equivalence is obtained by a construction (which is also exponential) that replaces the non-determinism of the functional VPT with deterministic look-ahead. This also yields an elegant and simple characterization of functional VPT.

3. Equivalence of functional $\mathrm{VPT}_{\mathrm{la}}$ is, as for $\mathrm{VPT}$, EXPTIME-C.

Therefore even though $\mathrm{VPT}_{\mathrm{la}}$ are exponentially more succinct than VPT, testing equivalence of functional $\mathrm{VPT}_{\mathrm{la}}$ is not harder than for functional VPT. This is done in two steps. First one checks equivalence of the domains. Then one checks that the union of the two transducers is still functional. We show that testing functionality is EXPTIME-C for $\mathrm{VPT}_{\mathrm{la}}$ : get rid of the look-aheads with an exponential blow-up and test in PTIME the functionality of the constructed VPT. To verify that the domains are equivalent, the naive technique (removing the look-aheads and then verifying the mutual inclusion of the domains) yields a doubly exponential algorithm. Instead, we show that the domains of $\mathrm{VPT}_{\mathrm{la}}$ are linearly reducible to alternating top-down tree automata. Testing the equivalence of such automata can be done in EXPTimE [3].

4. functional VPT and unambiguous VPT are equally expressive.

As an application of look-aheads, we show that a nice consequence of the constructions involved in contributions 1 and 3 is that functional VPT are effectively characterized by unambiguous VPT. This result was already known for finite-state transducers $[4,11,5]$ and here we extend it to VPT with rather simple constructions based on the concept of look-aheads. This characterization of functional finite-state transducers has been generalized to $k$-valued and $k$-ambiguous finite-state transducers [13] and recently with a better upper-bound [10] based on lexicographic decomposition of transducers.

Finally, we discuss slightly different look-aheads. First, we consider look-aheads that are allowed to inspect the whole current prefix until the end. We show this does not add expressivity nor succinctness. Second, we show that restricting the look-ahead to the current prefix in between the current call and its matching return is not sufficient to have a characterization of functional VPT by deterministic VPT $\mathrm{l}_{\mathrm{la}}$. All these results are new indications that the class of VPT is robust, and they show that the class of VPT la is interesting in itself.

Related Works Regular look-aheads have been mainly considered for classes of tree transducers, where a transition can be fired provided the current subtree belongs to some regular tree language. For instance, regular look-aheads have been added to topdown (ranked) tree transducers in order to obtain a robust class of tree transducers that enjoys good closure properties wrt composition [6], or to macro tree transducers 
(MTT) [7]. For top-down tree transducers, adding regular look-ahead strictly increases their expressive power while MTT are closed by regular look-ahead [7].

Trees over an alphabet $\Sigma$ can be linearized as well-nested words over the structured alphabet $\Sigma_{c}=\left\{c_{a} \mid a \in \Sigma\right\}, \Sigma_{r}=\left\{r_{a} \mid a \in \Sigma\right\}$. It is well-known that unranked trees can be represented by binary trees via the classical first-child next-sibling encoding (fcns). Top-down (ranked) tree transducers can thus be used as unranked tree transducers on fcns encodings of unranked trees. Inspecting a subtree in the fncs encoding corresponds to inspecting the first subtree and its next-sibling subtrees in an unranked tree, which in turn corresponds to inspecting the current longest well-nested prefix in their linearization. However as explained in [8], top-down tree transducers and VPT are incomparable: top-down tree transducers can copy subtrees while VPT cannot, and VPT support concatenation of tree sequences while top-down tree transducers cannot.

Modulo those encodings, MTT subsume VPT [8] and as we said before, there is a correspondence between the two notions of look-aheads, for VPT and MTT respectively. However it is not clear how to derive our results on closure by look-aheads from the same result on MTT, as the latter highly relies on parameters and it would require back-and-forth encodings between the two models. The direct construction we give in this paper is self-contained and allows one to derive the characterization of functional VPT as unambiguous VPT by a careful analysis of the construction.

\section{Visibly Pushdown Languages and Transductions}

All over this paper, $\Sigma$ denotes a finite alphabet partitioned into two disjoint sets ${ }^{2} \Sigma_{c}$, $\Sigma_{r}$, denoting respectively the call and return alphabets. We denote by $\Sigma^{*}$ the set of (finite) words over $\Sigma$ and by $\epsilon$ the empty word. The length of a word $u$ is denoted by $|u|$. The set of well-nested words $\Sigma_{\mathrm{wn}}^{*}$ is the smallest subset of $\Sigma^{*}$ such that $\epsilon \in \Sigma_{\mathrm{wn}}^{*}$ and for all $c \in \Sigma_{c}$, all $r \in \Sigma_{r}$, all $u, v \in \Sigma_{\mathrm{wn}}^{*}$, cur $\in \Sigma_{\mathrm{wn}}^{*}$ and $u v \in \Sigma_{\mathrm{wn}}^{*}$.

A visibly pushdown automaton (VPA) [1] on finite words over $\Sigma$ is a tuple $A=$ $(Q, I, F, \Gamma, \delta)$ where $Q$ is a finite set of states, $I \subseteq Q$ the set of initial states, $F \subseteq Q$ the set of final states, $\Gamma$ the (finite) stack alphabet, and $\delta=\delta_{c} \uplus \delta_{r}$ where $\delta_{c} \subseteq Q \times$ $\Sigma_{c} \times \Gamma \times Q$ are the call transitions, $\delta_{r} \subseteq Q \times \Sigma_{r} \times \Gamma \times Q$ are the return transitions.

On a call transition $\left(q, a, \gamma, q^{\prime}\right) \in \delta_{c}, \gamma$ is pushed onto the stack and the control goes from $q$ to $q^{\prime}$. On a return transition $\left(q, a, \gamma, q^{\prime}\right) \in \delta_{r}, \gamma$ is popped from the stack.

A configuration of a VPA is a pair $(q, \sigma) \in Q \times \Gamma^{*}$. A run of $T$ on a word $u=$ $a_{1} \ldots a_{l} \in \Sigma^{*}$ from a configuration $(q, \sigma)$ to a configuration $\left(q^{\prime}, \sigma^{\prime}\right)$ is a finite sequence $\rho=\left\{\left(q_{k}, \sigma_{k}\right)\right\}_{0 \leq k \leq l}$ such that $q_{0}=q, \sigma_{0}=\sigma, q_{l}=q^{\prime}, \sigma_{l}=\sigma^{\prime}$ and for each $1 \leq$ $k \leq l$, there exists $\gamma_{k} \in \Gamma$ such that either $\left(q_{k-1}, a_{k}, \gamma_{k}, q_{k}\right) \in \delta_{c}$ and $\sigma_{k}=\sigma_{k-1} \gamma_{k}$ or $\left(q_{k-1}, a_{k}, \gamma_{k}, q_{k}\right) \in \delta_{r}$ and $\sigma_{k-1}=\sigma_{k} \gamma_{k}$. The run $\rho$ is accepting if $q_{0} \in I, q_{l} \in F$ and $\sigma_{0}=\sigma_{l}=\perp^{3}$. A word $w$ is accepted by $A$ if there exists an accepting run of $A$ over w. $L(A)$, the language of $A$, is the set of words accepted by $A$. A language $L$ over $\Sigma$ is a visibly pushdown language if there is a VPA $A$ over $\Sigma$ such that $L(A)=L$. Finally, a

\footnotetext{
${ }^{2}$ In contrast to [1], we do not consider internal symbols $i$, as they can be simulated by a (unique) call $c_{i}$ followed by a (unique) return $r_{i}$. All our results extend trivially to alphabets with internal symbols. We make this assumption to simplify notations.

${ }^{3}$ Note that, in contrast to [1] and to ease notations, we do not allow return transition on $\perp$ and we require the final stack to be empty. This implies that all accepted words are well-nested.
} 
VPT is unambiguous if there is at most one accepting run per input word. In particular, any unambiguous VPT is functional. Unambiguity can be checked in PTIME [8].

As finite-state transducers extend finite-state automata with outputs, visibly pushdown transducers extend visibly pushdown automata with outputs [8]. To simplify notations, we suppose that the output alphabet is $\Sigma$, but our results still hold for an arbitrary output alphabet. Informally, the stack behavior of a VPT is similar to the stack behavior of visibly pushdown automata (VPA). On a call symbol, the VPT pushes a symbol on the stack and produces some output word (possibly empty and not necessarily wellnested), on a return symbol, it must pop the top symbol of the stack and produce some output word (possibly empty) and on an internal symbol, the stack remains unchanged and it produces some output word.

Definition 1. A visibly pushdown transducer (VPT) on finite words over $\Sigma$ is a tuple $T=(Q, I, F, \Gamma, \delta)$ where $Q$ is a finite set of states, $I \subseteq Q$ is the set of initial states, $F \subseteq Q$ the set of final states, $\Gamma$ is the stack alphabet, $\delta=\delta_{c} \uplus \delta_{r}$ the (finite) transition relation, with $\delta_{c} \subseteq Q \times \Sigma_{c} \times \Sigma^{*} \times \Gamma \times Q, \delta_{r} \subseteq Q \times \Sigma_{r} \times \Sigma^{*} \times \Gamma \times Q$.

Configurations and runs are defined similarly as VPA. Given a word $u=a_{1} \ldots a_{l} \in$ $\Sigma^{*}$ and a word $v \in \Sigma^{*}, v$ is an output of $u$ by $T$ if there exists an accepting run $\rho=\left\{\left(q_{k}, \sigma_{k}\right)\right\}_{0 \leq k \leq l}$ on $u$ and $l$ words $v_{1}, \ldots, v_{l}$ such that $v=v_{1} \ldots v_{l}$ and for all $0 \leq k<l$, there is a transition of $T$ from $\left(q_{k}, \sigma_{k}\right)$ to $\left(q_{k+1}, \sigma_{k+1}\right)$ that produces the output $v_{k+1}$ on input letter $a_{k+1}$. We write $(q, \sigma) \stackrel{u / v}{\longrightarrow}\left(q^{\prime}, \sigma^{\prime}\right)$ when there exists a run on $u$ from $(q, \sigma)$ to $\left(q^{\prime}, \sigma^{\prime}\right)$ producing $v$ as output. A transducer $T$ defines the binary word relation $\llbracket T \rrbracket=\left\{(u, v) \mid \exists q \in I, q^{\prime} \in F,(q, \perp) \stackrel{u / v}{\longrightarrow}\left(q^{\prime}, \perp\right)\right\}$.

A transduction is a binary relation $R \subseteq \Sigma^{*} \times \Sigma^{*}$. We say that a transduction $R$ is a VPT-transduction if there exists a VPT $T$ such that $R=\llbracket T \rrbracket$. A transduction $R$ is functional if for all $u \in \Sigma^{*}$, there exists at most one $v \in \Sigma^{*}$ such that $(u, v) \in R$. A VPT $T$ is functional if $\llbracket T \rrbracket$ is functional. Two transducers $T_{1}, T_{2}$ are equivalent if $\llbracket T_{1} \rrbracket=\llbracket T_{2} \rrbracket$. It is known [8] that functionality is decidable in PTIME for VPT and equivalence of functional VPT is EXPTIME-C.

The class of functional VPT is denoted by fVPT. For any input word $u \in \Sigma^{*}$, we denote by $R(u)$ the set $\{v \mid(u, v) \in R\}$. Similarly, for a VPT $T$, we denote by $T(u)$ the set $\llbracket T \rrbracket(u)$. If $R$ is functional, we confound $R(u)$ (which is at most of cardinality 1 ) and the unique image of $u$ if it exists. The domain of $T$ (denoted by $\operatorname{Dom}(T)$ ) is the domain of $\llbracket T \rrbracket$. Note that the domain of $T$ contains only well-nested words, which is not necessarily the case of the codomain.

Example 1. Let $\Sigma_{c}=\{c, a\}, \Sigma_{r}=\{r\}$ be the call and return symbols of the alphabet. The following VPT $T$ transforms a word as follows: (i) $a$ and $r$ are mapped to $a$ and $r$ respectively; (ii) $c$ is mapped either to $c$ if no $a$ appears in the longest well-nested word starting just after $c$, and to $a$ if an $a$ appears. E.g. ccrarrcr is mapped to acrarrcr, and cccarrcrcarrrr to aaaarrcraarrrr.

The VPT $T=(Q, I, F, \Gamma, \delta)$ is defined by $Q=\left\{q, q_{a}\right\}, I=\{q\}, F=Q, \Gamma=$ $\{(a, a),(a, \neg a),(\neg a, a),(\neg a, \neg a),(-, a)\}$ and $\delta$ contains the following transitions:

$$
\begin{array}{cccc}
q & \stackrel{c / c,(\neg a, \neg a)}{\longrightarrow} q & q \stackrel{c / a,(a, \neg a)}{\longrightarrow} q & q \stackrel{a / a,(-, a)}{\longrightarrow} q \\
q_{a} \stackrel{c / c,(\neg a, a)}{\longrightarrow} q & q_{a} \stackrel{c / a,(a, a)}{\longrightarrow} q & q_{a} \stackrel{a / a,(-, a)}{\longrightarrow} q \\
q \stackrel{r / r,(\neg a, \neg a)}{\longrightarrow} q & q \stackrel{r / r,(a, \neg a)}{\longrightarrow} q_{a} & q \stackrel{r / r,(-, a)}{\longrightarrow} q_{a} \\
q_{a} \stackrel{r / r,(a, \neg a)}{\longrightarrow} q_{a} & q_{a} \stackrel{r / r,(a, a)}{\longrightarrow} q_{a} & q_{a} \stackrel{r / r,(-, a)}{\longrightarrow} q_{a}
\end{array}
$$


If $T$ is in state $q_{a}$ it means that there is an $a$ in the longest well-nested word that ends at the current position, otherwise it is in state $q$. Consider an element $(\alpha, \beta)$ of the stack. If $\alpha=a$, respectively $\neg a$, it means that the call was a $c$ and the automaton translated it into an $a$, respectively translated into a $c$, i.e. it guesses there is an $a$, respectively there is no $a$, in the well-nested word starting just after $c$. If $\alpha=-$ then the call was an $a$. The second component $\beta$, carry over the fact that there is or not an $a$ in the well nested word ending at the current call.

\section{VPT with Visibly Pushdown Look-Ahead}

Given a word $w$ over $\Sigma$ we denote by $\operatorname{pref}_{\mathrm{wn}}(w)$ the longest well-nested prefix of $w$. E.g. $\operatorname{pref}_{\mathrm{wn}}(c c r c r)=\epsilon$ and $\operatorname{pref}_{\mathrm{wn}}(c r c)=c r$. We define a VPT $T$ with visibly pushdown look-ahead (simply called look-ahead in the sequel) informally as follows. The lookahead is given by a VPA $A$ without initial state. On a call symbol $c, T$ can trigger the look-ahead from a state $p$ of the VPA (which depends on the call transition). The lookahead tests membership of the longest well-nested prefix of the current suffix (that starts by the letter $c$ ) to $L(A, p)$, where $(A, p)$ is the VPA $A$ with initial state $p$. If the prefix is in $L(A, p)$ then the transition of $T$ can be fired. When we consider nested words that encode trees, look-ahead correspond to inspecting the subtree rooted at the current node and all right sibling subtrees (in other words, the current hedge). Formally:

Definition 2. A VPT with look-ahead $\left(\mathrm{VPT}_{\mathrm{la}}\right)$ is a pair $T_{l a}=(T, A)$ where $A$ is a VPA $A=\left(Q^{l a}, F^{l a}, \Gamma^{l a}, \delta^{l a}\right)$ without initial state and $T$ is a tuple $T=\left(Q, q_{0}, F, \Gamma, \delta\right)$ such that $Q$ is a finite set of states, $q_{0} \in Q$ is an initial state, $F \subseteq Q$ is a set of final states, $\Gamma$ is a stack alphabet, and $\delta=\delta_{c} \uplus \delta_{r}$ is a transition relation such that $\delta_{c} \subseteq Q \times \Sigma_{c} \times \Sigma^{*} \times Q^{l a} \times \Gamma \times Q$ and $\delta_{r} \subseteq Q \times \Sigma_{r} \times \Sigma^{*} \times \Gamma \times Q$.

Let $u \in \Sigma^{*}$. A run of $T_{l a}$ on $u=a_{1} \ldots a_{l}$ is a sequence of configurations $\rho=$ $\left\{\left(q_{k}, \sigma_{k}\right)\right\}_{0 \leq k \leq l}$ such that there exist $\gamma \in \Gamma$ and $v_{k+1} \in \Sigma^{*}$ such that $(i)$ if $a_{k+1} \in$ $\Sigma_{r}$, then $\sigma_{k+1} \gamma=\sigma_{k}$ and $\left(q_{k}, a_{k+1}, v_{k+1}, \gamma, q_{k+1}\right) \in \delta_{r} ;(i i)$ if $a_{k+1} \in \Sigma_{c}$, then $\sigma_{k+1}=\sigma_{k} \gamma$, and there exists $p \in Q^{l a}$ such that $\left(q_{k}, a_{k+1}, v_{k+1}, p, \gamma, q_{k+1}\right) \in \delta_{c}$ and $\operatorname{pref}_{\mathrm{wn}}\left(a_{k+1} \ldots a_{l}\right) \in L(A, p)$. The run $\rho$ is accepting if $\sigma_{0}=\sigma_{l}=\perp$ and $q_{l} \in F$. The word $v_{1} \ldots v_{l}$ is an output of $u$.

The $\mathrm{VPT}_{\text {la }} T_{l a}$ is deterministic if for all transitions $\left(q, c, v_{1}, p_{1}, \gamma_{1}, q_{1}\right) \in \delta_{c}$ and $\left(q, c, v_{2}, p_{2}, \gamma_{2}, q_{2}\right) \in \delta_{c}$, if $v_{1} \neq v_{2}$ or $\gamma_{1} \neq \gamma_{2}$ or $q_{1} \neq q_{2}$ or $p_{1} \neq p_{2}$, then $L\left(A, p_{1}\right) \cap$ $L\left(A, p_{2}\right)=\varnothing$; and for all transitions $\left(q, r, v_{1}, \gamma_{1}, q_{1}\right) \in \delta_{r}$ and $\left(q, r, v_{2}, \gamma_{2}, q_{2}\right) \in \delta_{r}$ we have $v_{1}=v_{2}, \gamma_{1}=\gamma_{2}$ and $q_{1}=q_{2}$. Note that deciding whether some VPT $\mathrm{P}_{\text {la }}$ is deterministic can be done in PTIME. One has to check that for each state $q$ and each call symbol $c$, the VPL guarding the transition from state $q$ and reading $c$ are pairwise disjoint. The number of states of a $\mathrm{VPT}_{\text {la }}$ is the number of states of the transducer plus the number of states of the look-ahead.

Example 2. A $\mathrm{VPT}_{\mathrm{la}}$ is represented in Figure 1. The look-ahead automaton is depicted on the right, while the transducer in itself is on the left. It defines the transduction of Example 1. When starting in state $q_{a}$, respectively $q_{\neg a}$, the look-ahead automaton accepts well-nested words that contains an $a$, respectively does not contain any $a$. When starting in state $q_{f}$ it accepts any well-nested word. The transducer rewrites $c$ symbols 

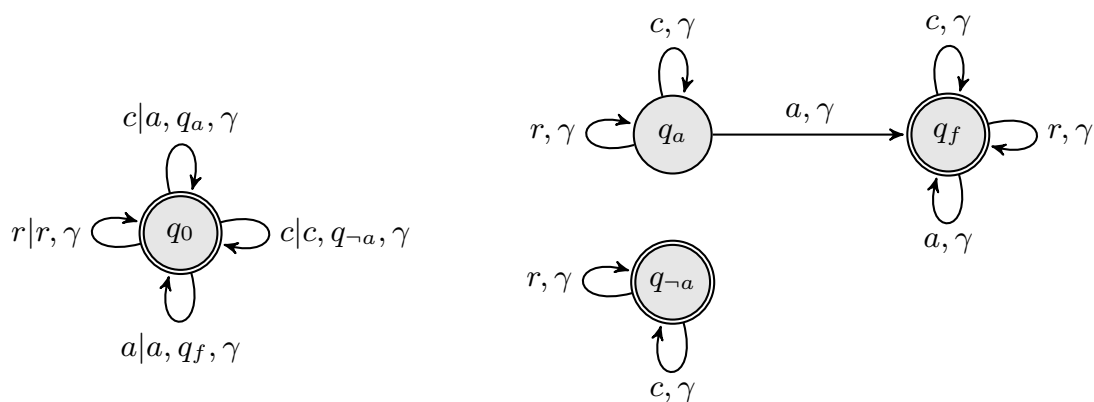

Fig. 1. A $\mathrm{VPT}_{\mathrm{la}}$ (left) and its look-ahead (right) on $\Sigma_{c}=\{c, a\}$ and $\Sigma_{r}=\{r\}$

into $a$ if the well-nested word starting at $c$ contains an $a$ (transition on the top), otherwise it just copy a $c$ (transition on the right). This is achieved using the $q_{a}$ and $q_{\neg a}$ states of the look-ahead automaton. Other input symbols, i.e. $a$ and $r$, are just copied to the output (left and bottom transitions).

The next theorem states that adding look-aheads to VPT does not add expressiveness. The main difficulty is to simulate an unbounded number of look-aheads at the same time. Indeed, a look-ahead is triggered at each call and is life until the end of the wellnested subword starting at this call. We use summaries [1] to handle look-aheads that started at a strictly less deeper nesting level and a subset construction for those that started at the same nesting level.

Theorem 1. For all $\mathrm{VPT}_{\text {la }} T_{\text {la }}$ with $n$ states, one can construct an equivalent $\mathrm{VPT} T^{\prime}$ with $O\left(n 2^{n^{2}+1}\right)$ states. Moreover, if $T_{l a}$ is deterministic, then $T^{\prime}$ is unambiguous.

Proof. Let $T_{l a}=(T, A)$ with $T=\left(Q, q_{0}, F, \Gamma, \delta\right)$ and $A=\left(Q^{l a}, F^{l a}, \Gamma^{l a}, \delta^{l a}\right)$. We construct $T^{\prime}=\left(Q^{\prime}, q_{0}^{\prime}, F^{\prime}, \Gamma^{\prime}, \delta^{\prime}\right)$ as follows (where $I d_{Q^{l a}}$ denotes the identity relation on $\left.Q^{l a}\right)$ :

$$
\begin{array}{ll}
Q^{\prime}=Q \times 2^{Q^{l a} \times Q^{l a}} \times 2^{Q^{l a}} & q_{0}^{\prime}=\left(q_{0}, I d_{\left.Q^{l a}, \varnothing\right)}\right) \\
F^{\prime}=\left\{(q, R, L) \in Q^{\prime} \mid q \in F, L \subseteq F^{l a}\right\} & \Gamma^{\prime}=\Gamma \times 2^{Q^{l a} \times Q^{l a}} \times 2^{Q^{l a}} \times \Sigma_{c}
\end{array}
$$

The transducers $T^{\prime}$ simulates $T$ and its running look-aheads. A state of $T^{\prime}$ is a triple $(q, R, L)$. The first component is the state of $T$. The second and third components are used to simulate the running look-aheads. When taking a call $c, T^{\prime}$ non-deterministically chooses a new look-ahead triggered by $T$. This look-ahead is added to all running lookaheads that started at the same nesting level. $T^{\prime}$ ensures that the run will fail if the longest well-nested prefix starting at $c$ is not in the language of the triggered lookahead. The $L$ component contains the states of all running look-aheads triggered at the current nesting level. The $R$ component is the summary (see [1]) necessary to update the $L$-component. When reading a call the $L$ component is put on the stack. When reading a return, $T^{\prime}$ must check that all look-ahead states in $L$ are final, i.e. $T^{\prime}$ ensures that the chosen look-ahead are successful.

After reading a well-nested word $w$ if $T^{\prime}$ is in state $(q, R, L)$, with $q \in Q, R \subseteq$ $Q^{l a} \times Q^{l a}$ and $L \subseteq Q^{l a}$, we have the following properties. The pair $\left(p, p^{\prime}\right) \in R$ iff there exists a run of $A$ from $p$ to $p^{\prime}$ on $w$. If some $p^{\prime \prime}$ is in $L$, there exists a run of a look-ahead that started when reading a call symbol of $w$ at depth 0 which is now in state $p^{\prime \prime}$. Conversely, for all look-aheads that started when reading a call symbol of $w$ at depth 0 , there exists a state $p^{\prime \prime} \in L$ and a run of this look-ahead that is in state $p^{\prime \prime}$. 


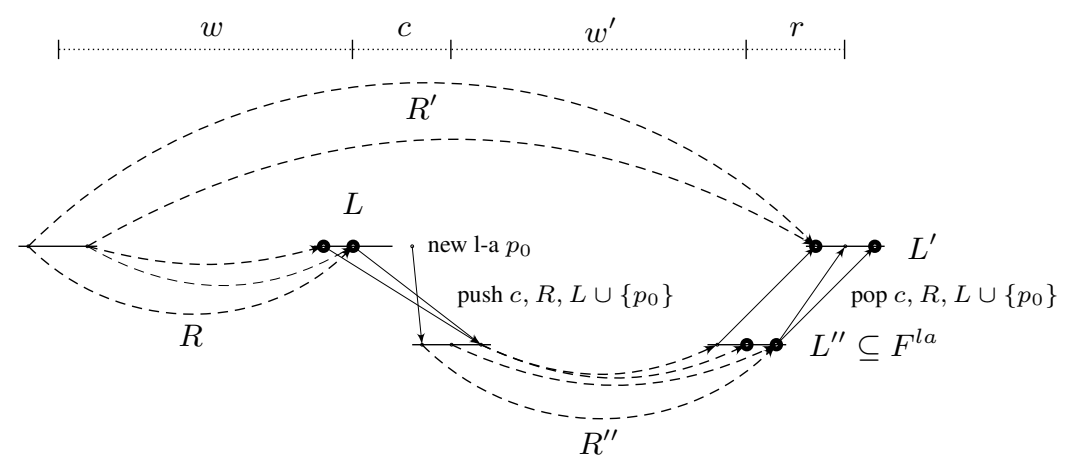

Fig. 2 .

Let us consider a word $w c w^{\prime} r$ for some well-nested words $w, w^{\prime}$ (depicted on Fig. 2 ). Assume that $T^{\prime}$ is in state $(q, R, L)$ after reading $w$ (on the figure, the relation $R$ is represented by dashed arrows and the set $L$ by big points, and other states by small points). We do not represent the $T$-component of the states on the figure but rather focus on $R$ and $L$. The information that we push on the stack when reading $c$ is the necessary information to compute a state $\left(q^{\prime}, R^{\prime}, L^{\prime}\right)$ of $T^{\prime}$ reached after reading $w c w^{\prime} r$. After reading the call symbol $c$, we go in state $\left(q^{\prime}, I d_{Q^{l a}}, \varnothing\right)$ and produce the output $v$ for some $q^{\prime}, v$ such that $q \stackrel{c \mid v, p_{0}, \gamma}{\longrightarrow} q^{\prime} \in \delta_{c}$, where $p_{0} \in Q^{l a}$ is the starting state of a new look-ahead. Note that determinism of $T$ is preserved. On the stack we put the tuple $\left(\gamma, R, L \cup\left\{p_{0}\right\}, c\right)$ where $\gamma, R, L, p_{0}, c$ have been defined before.

Now, suppose that after reading $w c w^{\prime}$ the transducer $T^{\prime}$ is in state $\left(q^{\prime \prime}, R^{\prime \prime}, L^{\prime \prime}\right)$. It means that $T$ is in state $q^{\prime \prime}$ after reading $w c w^{\prime}$, and $\left(p, p^{\prime}\right) \in R^{\prime \prime}$ iff there exists a run of $A$ from $p$ to $p^{\prime}$ on $w^{\prime}$, and $L^{\prime \prime}$ is some set of states reached by the look-aheads that started at the same depth as $w^{\prime}$. Therefore we first impose that any transition from $\left(q^{\prime \prime}, R^{\prime \prime}, L^{\prime \prime}\right)$ reading $r$ must satisfy $L^{\prime \prime} \subseteq F^{l a}$. Clearly, $R^{\prime}$ can be constructed from $c$, $R$ and $R^{\prime \prime}$. Finally, $L^{\prime}$ is a set which satisfies for all $p \in L \cup\left\{p_{0}\right\}$, there exists $p^{\prime} \in L^{\prime}$ such that there exists a run of $A$ from $p$ to $p^{\prime}$ on $c w^{\prime} r$. If such an $L^{\prime}$ does not exist, there is no transition on $r$. The set $L^{\prime}$ can be constructed from $L \cup\left\{p_{0}\right\}$ and $R^{\prime \prime}$.

We now define the transitions formally. First, for all $q, R, L, c, \gamma$, we have:

$$
(q, R, L) \stackrel{c \mid u,\left(\gamma, R, L \cup\left\{p_{0}\right\}, c\right)}{\longrightarrow}\left(q^{\prime}, I d_{Q^{l a}}, \varnothing\right) \in \delta_{c}^{\prime} \text { whenever } q \stackrel{c \mid u, p_{0}, \gamma}{\longrightarrow} q^{\prime} \in \delta_{c}
$$

Then, for all $R, L, r, \gamma, q^{\prime \prime}, R^{\prime \prime}, L^{\prime \prime}, q^{\prime}, R^{\prime}, L^{\prime}$ we have:

$$
\left(q^{\prime \prime}, R^{\prime \prime}, L^{\prime \prime}\right) \stackrel{r \mid u,(\gamma, R, L, c)}{\longrightarrow}\left(q^{\prime}, R^{\prime}, L^{\prime}\right) \in \delta_{r}^{\prime}
$$

if the following conditions hold:

(i) $q^{\prime \prime} \stackrel{r \mid u, \gamma}{\longrightarrow} q^{\prime} \in \delta_{r},\left(\right.$ ii) $L^{\prime \prime} \subseteq F^{l a}$

(iii) $R^{\prime}=\left\{\left(p, p^{\prime}\right) \mid \exists s \stackrel{c, \gamma}{\longrightarrow} s^{\prime} \in \delta_{c}^{l a} \cdot \exists\left(s^{\prime}, s^{\prime \prime}\right) \in R^{\prime \prime} \cdot(p, s) \in R\right.$ and $\left.s^{\prime \prime} \stackrel{r, \gamma}{\longrightarrow} p^{\prime} \in \delta_{r}^{l a}\right\}$

(iv) for all $p \in L$, there exist $p^{\prime} \in L^{\prime}, \gamma \in \Gamma, s, s^{\prime} \in Q^{l a}$ such that $\left(s, s^{\prime}\right) \in R^{\prime \prime}$, $p \stackrel{c, \gamma}{\longrightarrow} s \in \delta_{c}^{l a}, s^{\prime} \stackrel{r, \gamma}{\longrightarrow} p^{\prime} \in \delta_{r}^{l a}$.

The proof of correctness is sketched in Appendix. If $T$ is deterministic, then $T^{\prime}$ is unambigous. Indeed, it is deterministic on return transitions. If there are two possible 
transitions $q \stackrel{c \mid u_{1}, p_{1}, \gamma_{1}}{\longrightarrow} q_{1}$ and $q \stackrel{c \mid u_{2}, p_{2}, \gamma_{2}}{\longrightarrow} q_{2}$ on a call symbol $c$, as $T$ is deterministic, we know that either the look-ahead starting in $p_{1}$ or the look-ahead starting in $p_{2}$ will fail. In $T^{\prime}$, there will be two transitions that will simulate both look-aheads respectively, and therefore at least one continuation of the two transitions will fail as well. Therefore there is at most one accepting computation per input word in $T$.

Proposition 1. Let $\Sigma$ be a finite alphabet with at least two letters. There exists a family of transductions $\left(T_{n}\right)_{n}$ over $\Sigma$ such that for all $n \geq 0, T_{n}$ is definable by a $\mathrm{VPT}_{\text {la }}$ with $O(n)$ states and any VPT defining $L_{n}$ has at least $O\left(2^{n}\right)$ states.

Proof (sketch). We show that the result already holds for finite state automata with regular look-ahead, which yields a construction for VPT la. VPT on flat words (in $\left(\Sigma_{c} \Sigma_{r}\right)^{*}$ ) can indeed simulate finite state automata (in that case the stack is useless). For all $n$, we define the language $L_{n}=\{v u v|| v \mid=n\}$. We show that $L_{n}$ can be recognized by a finite state automaton with regular look-ahead with $O(n)$ states while without regular look-ahead any automaton would need at least $|\Sigma|^{n}$ states.

\section{Functional VPT and VPT}

While there is no known syntactic restriction on VPT that captures all functional VPT, we show that the class of deterministic VPT la captures all functional VPT. As there may be an unbounded number of accepting runs, the equivalent $\mathrm{VPT}_{\text {la }}$ has to choose only one of them by using look-aheads. This is done by ordering the states and extending this order to runs. The main difficulty is to cope with nesting. Indeed, when the transducer enters an additional level of nesting, its look-ahead cannot inspect the entire suffix but is limited to the current nesting level. When reading a call, choosing (thanks to some look-ahead) the smallest run on the current well-nested prefix is not correct because it may not be possible to extend this run to an accepting run on the entire word. Therefore the transducer has to pass some information from one to the next level of nesting about the chosen global run.

Theorem 2. For all functional VPT $T$, one can construct a deterministic VPT la $T_{l a}$ with exponentially many more states such that $\llbracket T \rrbracket=\llbracket T_{l a} \rrbracket$.

Proof. It is clear that deterministic VPT la are functional VPT. For the converse, we order the states of $T$ and use look-aheads to choose the smallest runs wrt to an order on runs that depends on the structure of the word. Let $T=\left(Q, q_{0}, F, \Gamma, \delta\right)$ be a functional VPT. Wlog we assume that for all $q, q^{\prime} \in Q$, all $\alpha \in \Sigma$, there is at most one $u \in \Sigma^{*}$ and one $\gamma \in \Gamma$ such that $\left(q, \alpha, u, \gamma, q^{\prime}\right) \in \delta$. A transducer satisfying this property can be obtained by duplicating the states with transitions, i.e. by taking the set of states $Q \times \Delta$.

We construct an equivalent deterministic $\operatorname{VPT}_{\text {la }}\left(T^{\prime}, A\right)$ where $T^{\prime}=\left(Q^{\prime}, q_{0}, F^{\prime}, \Gamma^{\prime}, \delta^{\prime}\right)$ with $Q^{\prime}=\left\{q_{0}\right\} \cup Q^{2}, F^{\prime}=F \times Q$ if $q_{0} \notin F$ otherwise $F^{\prime}=(F \times Q) \cup\left\{q_{0}\right\}$. The look-ahead $A$ is defined later. Before defining $\delta^{\prime}$ formally, let us explain it informally. There might be several accepting runs on an input word $w$, each of them producing the same output, as $T$ is functional. To ensure determinism, $T^{\prime}$ has to choose exactly one transition when reading a symbol. The idea is to order the states by a total order $<_{Q}$ and 
to extend this order to runs. The look-ahead will be used to choose the next transition of $T$ that has to be fired, so that the choice will ensure that $T$ follows the smallest accepting run on $w$. However the look-ahead can only visit the current longest well-nested prefix, and not the entire word. Therefore the "parent" of the call $c$ has to pass some information about the global run to its child $c$. In particular, when $T^{\prime}$ is in state $\left(q, q^{\prime}\right)$ for some state $q^{\prime}$, it means that $T$ is in state $q$ and the state reached after reading the last return symbol of the longest-well nested current prefix must be $q^{\prime}$.

Consider a word of the form $w=c_{1} w_{1} r_{1} w_{2} c_{3} w_{3} r_{3}$ where $w_{i}$ are well-nested, depicted on Fig. 3. Suppose that before evaluating $w, T^{\prime}$ is in state $\left(q_{1}, q_{3}\right)$. It means that the last transition $T$ has to fire when reading $r_{3}$ has a target state $q_{3}$. When reading the call symbol $c_{1}, T^{\prime}$ uses a look-ahead to determine the smallest triple of states $\left(q_{1}^{\prime}, q_{2}^{\prime}, q_{2}\right)$ such that there exists a run on $w$ that starts in $q_{1}$ and such that after reading $c_{1}$ it is in state $q_{1}^{\prime}$, before reading $r_{1}$ it is in state $q_{2}^{\prime}$, after reading $r_{1}$ it is in state $q_{2}$ and after reading $r_{3}$ it is in state $q_{3}$. Then, $T^{\prime}$ fires the call transition on $c_{1}$ that with source and target states $q_{1}$ and $q_{1}^{\prime}$ respectively (it is unique by hypothesis), put on the stack the states $\left(q_{2}, q_{3}\right)$ and passes to $w_{1}$ (in the state) the information that the chosen run on $w_{1}$ terminates by the state $q_{2}^{\prime}$, i.e. it goes to the state $\left(q_{1}^{\prime}, q_{2}^{\prime}\right)$. (see Fig. 3). On the figure, we do not explicit all the states and anonymous components are denoted by .. When reading $r_{1}, T^{\prime}$ pops from the stack the tuple $\left(\gamma, q_{2}, q_{3}\right)$ and therefore knows that the transition to apply on $r_{1}$ has target state $q_{2}$ and the transition to apply on $r_{3}$ has target state $q_{3}$. Then it passes $q_{3}$ to the current state.

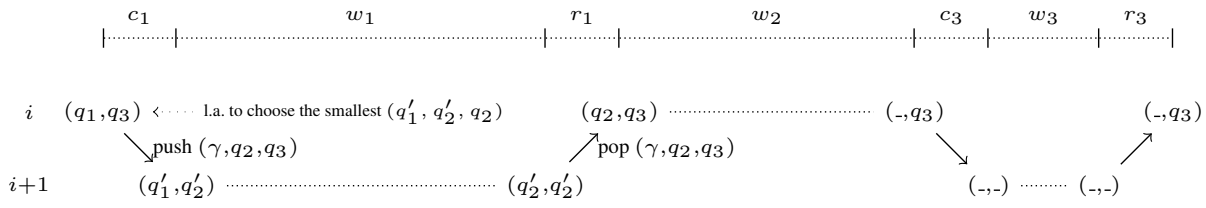

Fig. 3.

When the computation starts in $q_{0}$, we do not know yet what return transition has to be fired at the end of the hedge. This case can be easily treated separately by a lookahead on the first call symbol that determine the smallest 4-tuple of states $\left(q_{1}, q_{2}^{\prime}, q_{2}, q_{3}\right)$ which satisfies the conditions described before, but to simplify the proof, we assume that the VPT accepts only words of the form $c w r$, where $w$ is well-nested, so that one only needs to consider triples of states.

We now define the transition relation formally. Let $<$ be a total order on states, extended lexicographically to tuples. For all states $q_{1}, q_{1}^{\prime}, q_{2}^{\prime}, q_{2}, q_{3} \in Q$, it is easy to define a VPA $A_{q_{1}, q_{1}^{\prime}, q_{2}^{\prime}, q_{2}, q_{3}}$ whose size is polynomial in the size of $T$ that accepts a word $w$ iff it is of the form $c_{1} w_{1} r_{1} w_{3}$ where $w_{1}, w_{3}$ are well-nested and there exists a run of $T$ on $w$ that starts in state $q_{1}$ and is state $q_{1}^{\prime}$ after reading $c_{1}$, in state $q_{2}^{\prime}$ before reading $r_{1}$, in state $q_{2}$ after reading $r_{1}$ and in state $q_{3}$ after reading $w_{3}$. Note that if $w_{3}=\epsilon$ then if $q_{3} \neq q_{2}$, then $w \notin L\left(A_{q_{1}, q_{1}^{\prime}, q_{2}^{\prime}, q_{2}, q_{3}}\right)$. We denote by $\overline{A_{q_{1}, q_{1}^{\prime}, q_{2}^{\prime}, q_{2}, q_{3}}}$ the

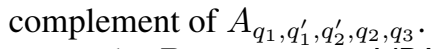

We let $B_{q_{1}, q_{1}^{\prime}, q_{2}^{\prime}, q_{2}, q_{3}}$ a VPA with initial state $p_{q_{1}, q_{1}^{\prime}, q_{2}^{\prime}, q_{2}, q_{3}}$ that defines the language:

$$
L\left(B_{q_{1}, q_{1}^{\prime}, q_{2}^{\prime}, q_{2}, q_{3}}\right)=L\left(A_{q_{1}, q_{1}^{\prime}, q_{2}^{\prime}, q_{2}, q_{3}}\right) \cap \bigcap_{\begin{array}{c}
\left(s_{1}, s_{2}^{\prime}, s_{2}\right) \in Q^{3} \\
\left(s_{1}, s_{2}^{\prime}, s_{2}\right)<\left(q_{1}, q_{2}^{\prime}, q_{2}\right)
\end{array}} L\left(\overline{A_{q_{1}, s_{1}, s_{2}^{\prime}, s_{2}, q_{3}}}\right)
$$


Such a VPA exists as VPA are closed by intersection and complement. Its size however may be exponential in $|Q|$. We define the look-ahead VPA as the union of all those VPA, $A_{l a}=\biguplus B_{q_{1}, q_{1}^{\prime}, q_{2}^{\prime}, q_{2}, q_{3}}$. We now define the call and return transitions of $T^{\prime}$ as follows, for all $c \in \Sigma_{c}, r \in \Sigma_{r}, \gamma \in \Gamma, q_{1}, q_{1}^{\prime}, q_{2}^{\prime}, q_{3}, q \in Q, u \in \Sigma^{*}$ :

$$
\begin{gathered}
\left(q_{1}, q_{3}\right) \stackrel{c \mid u,\left(\gamma, q_{2}, q_{3}\right), p_{q_{1}, q_{1}^{\prime}, q_{2}^{\prime}, q_{2}, q_{3}}}{\stackrel{c \mid u,\left(\gamma, q_{3}, q_{3}\right), p_{q_{0}, q_{1}^{\prime}, q_{2}^{\prime}, q_{3}, q_{3}}}{\longrightarrow}}\left(q_{1}^{\prime}, q_{2}^{\prime}\right) \text { if }\left(q_{1}^{\prime}, q_{2}^{\prime}\right) \text { if }\left(q_{0} \stackrel{c \mid u, \gamma}{\longrightarrow} q_{1}^{\prime}\right) \in \delta_{c} \\
\left.q_{0} \stackrel{r \mid u, \gamma}{\longrightarrow} q_{1}^{\prime}\right) \in \delta_{c} \\
\left(q_{2}^{\prime}, q\right) \stackrel{r \mid u,\left(\gamma, q_{2}, q_{3}\right)}{\longrightarrow} \quad\left(q_{2}, q_{3}\right) \text { if }\left(q_{2}^{\prime} \stackrel{r \mid u, \gamma}{\longrightarrow} q_{2}\right) \in \delta_{r}
\end{gathered}
$$

The transducer $T^{\prime}$ is deterministic: return transitions are fully determined by the states $q_{2}^{\prime}, q_{2}, q_{3}$ and the input letter $r$ (by our first assumption there is at most one transition in $T$ from $q_{2}^{\prime}$ to $\left.q_{2}\right)$. For call transitions, suppose that from $\left(q_{1}, q_{3}\right)$ there are two possible look-aheads $p_{q_{1}, q_{1}^{\prime}, q_{2}^{\prime}, q_{2}, q_{3}}$ and $p_{q_{1}, s_{1}^{\prime}, s_{2}^{\prime}, s_{2}, s_{3}}$. By definition of the look-aheads, we have $L\left(B_{q_{1}, q_{1}^{\prime}, q_{2}^{\prime}, q_{2}, q_{3}}\right) \cap L\left(B_{q_{1}, q_{1}^{\prime}, q_{2}^{\prime}, q_{2}, q_{3}}\right)=\varnothing$. Moreover, there cannot be two transitions with the same look-ahead as transitions are fully determinied by $q_{1}, q_{3}, q_{2}, q_{2}^{\prime}, q_{1}^{\prime}$ (there is at most one call transition by our assumption with source and target states $q_{1}$ and $q_{1}^{\prime}$ respectively). A simple analysis of the complexity shows that the look-ahead $A$ has exponentially many more states than $T$ (the exponentiation comes from the complement in the definition of $\left.B_{q_{1}, q_{1}^{\prime}, q_{2}^{\prime}, q_{2}, q_{3}}\right)$.

This construction, followed by the construction of Theorem 1 that removes the lookaheads, yields a nice characterization of functional VPT:

Theorem 3. For all functional VPT $T$, one can effectively construct an equivalent unambigous VPT $T^{\prime}$.

\section{Functionality and Equivalence of $\mathrm{VPT}_{\text {la }}$}

In this section, we study the problems of functionality of $\mathrm{VPT}_{\mathrm{la}}$ and equivalence of functional $\mathrm{VPT}_{\mathrm{la}}$. In particular, we prove that while being exponentially more succinct than VPT, the equivalence of functional $\mathrm{VPT}_{\mathrm{la}}$ remains decidable in EXPTIME, as equivalence of functional VPT.

Theorem 4. Functionality of $\mathrm{VPT}_{\mathrm{la}}$ is EXPTIME-C, even for deterministic look-aheads.

Proof. For the EXPTIME upper-bound, we first apply Theorem 1 to remove the lookaheads. This results in a VPT possibly exponentially bigger. Then functionality can be tested in PTIME [8]. For the lower-bound, we reduce the problem of deciding emptiness of the intersection of $n$ deterministic top-down tree automata, which is known to be EXPTIME-C when $n$ is part of the input [3]. The full proof is in Appendix.

We know that the equivalence of two functional VPT is ExPTIME-C [8]. To decide the equivalence of two functional $\mathrm{VPT}_{\mathrm{la}}$, one can first remove the look-aheads, modulo an exponential blow-up, and use the procedure for VPT. This would yield a 2-EXPTIME procedure for the equivalence of functional $\mathrm{VPT}_{\text {la }}$. However, it is possible to decide it in EXPTIME:

Theorem 5. Equivalence of functional $\mathrm{VPT}_{\mathrm{la}}$ is EXPTIME-C, even if the transducers and the look-aheads are deterministic. 
Proof. The lower bound is obtained similarly as for functionality (see Appendix). For the upper-bound, in a first step we check equivalence of the respective domain of two $\mathrm{VPT}_{\mathrm{la}}$ (we show how to do it in EXPTIME). Then we check the equivalence as follows: transform each $\mathrm{VPT}_{\mathrm{la}}$ into an equivalent VPT with at most an exponential blow-up, take the union and verify (in PTIME) that the resulting VPT is still functional.

We now show how to check the equivalence of the domain of two $\mathrm{VPT}_{\text {la }}$. Let $T_{1}, T_{2}$ be two VPT $T_{\text {la }}$. Their domains are defined by VPA extended with visibly pushdown look-aheads: it suffices to project away the output words in $T_{1}$ and $T_{2}$ respectively. We do not formally define VPA with look-aheads, but they can be defined as VPT without considering output words. Let us denote $A_{1}, A_{2}$ the VPA with look-ahead that define $\operatorname{Dom}\left(T_{1}\right)$ and $\operatorname{Dom}\left(T_{2}\right)$ respectively. We show how to check $L\left(A_{1}\right)=L\left(A_{2}\right)$ in EXPTIME. The idea is to reduce the problem to equivalence of finite alternating tree automata, which is known to be in EXPTIME [3]. Well-nested words over the alphabet $\Sigma=\Sigma_{c} \uplus \Sigma_{r}$ can be translated as unranked trees over the alphabet $\tilde{\Sigma}=\Sigma_{c} \times \Sigma_{r}$. Those unranked trees can be again translated as binary trees via the classical first-child next-sibling encoding [3]. VPA over $\Sigma$ can be translated into equivalent top-down tree automata over first-child next-sibling encodings on $\tilde{\Sigma}$ of well-nested words over $\Sigma$ in PTIME [9]. Look-aheads of VPA inspect the longest well-nested prefix of the current suffix. This corresponds to subtrees in first-child next-sibling encodings of unranked trees. Therefore VPA with look-aheads can be translated into top-down tree automata with look-aheads that inspect the current subtree. Top-down tree automata with such look-aheads can be again translated into alternating tree automata: triggering a new look-ahead corresponds to a universal transition towards two states: the current state of the automaton and the initial state of the look-ahead. This again can be done in PTIME. Since equivalence of finite alternating tree automata is in EXPTIME [3], one gets an EXPTIME upper-bound for testing equivalence of the domains of two VPT $\mathrm{T}_{\mathrm{a}}$.

\section{Discussion and Conclusion}

Discussion We discuss several variants of visibly pushdown look-aheads. Instead of inspecting the longest well-nested current prefix, one could visit only the current wellnested prefix of the form $c w r$. This would correspond to the first subtree of the current hedge in encodings of unranked trees as well-nested words. While VPT would still be closed by such look-aheads, we would not have a correspondence between deterministic $\mathrm{VPT}_{\mathrm{la}}$ and functional VPT anymore. For instance, the class of transductions defined in Prop. 1 would not be definable by a deterministic VPT $\mathrm{V}_{\text {a }}$, although it is a functional transduction. In some sense, our definition of look-ahead is the minimal requirement to get the equivalence between deterministic $\mathrm{VPT}_{\mathrm{la}}$ and functional VPT.

One could also allow look-aheads on return transitions. Such look-aheads would inspect the longest well-nested prefix starting just after the current return symbol. This could be easily simulated by look-aheads on call transitions in PTIME, and it would preserve determinism. Therefore our results still hold in this setting.

Another way of adding look-aheads is to allow them to inspect the whole current suffix. Such look-aheads can be defined by visibly pushdown automata where return transitions on empty stack are allowed, as originally defined in [1]. The construction of Theorem 1 can be slightly modified to show that VPT are still closed by such lookaheads. The idea is to extend the states with a new component $L_{\perp} \subseteq Q^{l a}$ that corre- 
sponds to states of look-aheads that started at a deeper position than the current position. For those states we apply only return transitions on empty stack when reading a return symbol. See Appendix for a formal construction. Obviously, VPT with such look-aheads still satisfy the correspondence between functional VPT and deterministic VPT with look-ahead. However, the proof of the EXPTIME upper-bound for functional equivalence cannot be adapted as we cannot reduce the problem of testing equivalence of the domains to equivalence of alternating tree automata. We let the question of finding the exact complexity of functional equivalence for VPT with visibly pushdown look-ahead that inspect the whole suffix as future work.

Finally, note that most of our results also hold for VPA extended with visibly pushdown look-aheads: closure of VPA by visibly pushdown look-aheads, succinctness of VPA with look-aheads, and EXPTIME-completeness of equivalence of VPA with lookahead (even for deterministic VPA and look-aheads).

Future Work It is unknown whether $k$-valued VPT are equivalent to $k$-ambiguous $\mathrm{VPT}_{\mathrm{la}}$. This question seems more difficult than for functional VPT. Indeed, let us assume that the look-aheads can visit the whole suffix (this setting is more powerful but already allows us to give some insights on the difficulty of this problem). For functional VPT, when several transitions are possible, we know that any of them that can be completed into an accepting run can be triggered. Therefore one just has to order the transitions and, by using a look-ahead, trigger the smallest satisfying this property. For $k$-valued VPT, among a set of possible transitions it is necessary to choose for each output word (among at most $k$ output words) exactly one transition, in order to turn $k$-valuedness into $k$-ambiguity. It is not clear how to use look-aheads to make such choices.

\section{References}

1. R. Alur and P. Madhusudan. Visibly pushdown languages. In STOC, pages 202-211, 2004.

2. R. Alur and P. Madhusudan. Adding nesting structure to words. JACM, 56(3):1-43, 2009.

3. H. Comon, M. Dauchet, R. Gilleron, C. Löding, F. Jacquemard, D. Lugiez, S. Tison, and M. Tommasi. Tree automata techniques and applications, 2007.

4. S. Eilenberg. Automata, Languages, and Machines. Academic Press, Inc., 1974.

5. C. C. Elgot and J. E. Mezei. On relations defined by generalized finite automata. IBM Journal of Research and Development, 9:47-68, 1965.

6. J. Engelfriet. Top-down tree transducers with regular look-ahead. Mathematical Systems Theory, 10:289-303, 1977.

7. J. Engelfriet and H. Vogler. Macro tree transducers. JCSS, 31(1):71-146, 1985.

8. E. Filiot, J.-F. Raskin, P.-A. Reynier, F. Servais, and J.-M. Talbot. Properties of visibly pushdown transducers. In MFCS, pages 355-367, 2010.

9. O. Gauwin. Streaming Tree Automata and XPath. PhD thesis, Université Lille 1, 2009.

10. J. Sakarovitch and R. de Souza. Lexicographic decomposition of $\mathrm{k}$-valued transducers. TCS, 47(3):758-785, 2010.

11. M. P. Schützenberger. Sur les relations rationnelles entre monoides libres. TCS, 3(2):243$259,1976$.

12. S. Staworko, G. Laurence, A. Lemay, and J. Niehren. Equivalence of deterministic nested word to word transducers. In FCT, volume 5699 of LNCS, pages 310-322, 2009.

13. A. Weber. Decomposing finite-valued transducers and deciding their equivalence. SIAM Journal on Computing, 22(1):175-202, 1993. 


\section{A VPT with Visibly Pushdown Look-Ahead}

Proof (Correctness of the construction of Theorem 1). We sketch the proof of correctness of the construction. Let $w \in \Sigma^{*}$ such that $w$ is a prefix of well-nested word. We define $\operatorname{sh}(w)$ as the longest well-nested suffix of $w$, we call $s h(w)$ the subhedge of $w$. For instance, if $w=c_{1} c_{2} r_{2} c_{3} r_{3}$, then $s h(w)=c_{2} r_{2} c_{3} r_{3}$. However if $w=c_{1} c_{2}$, then $\operatorname{sh}(w)=\epsilon$.

First, one can check (e.g. by induction on the length of $w$ ) that the successive computations of the $R$ component of the state ensures that the following property holds: for all words $w \in \Sigma^{*}$ prefix of a well-nested word, if there is a run of $T^{\prime}$ from $q_{0}^{\prime}$ to $(q, R, L)$ on $w$, then for all $p, p^{\prime} \in Q^{l a},\left(p, p^{\prime}\right) \in R$ iff there is a run of $A$ on $\operatorname{sh}(w)$ from $p$ to $p^{\prime}$.

With this last property it is easy to show that the following property also holds: let $w=c_{1} w_{1} r_{1} c_{2} w_{2} r_{2} \ldots c_{n} w_{n} r_{n}$ where all $w_{i}$ are well-nested. A run of $T$ on $w$ will trigger a new look-ahead at each call $c_{i}$, all these look-ahead will still be 'live' until $r_{n}$. These look-aheads are simulated by the $L$ component of the state of $T^{\prime}$. If there is a run of $T$ on $w$, it means that all look-aheads accepts the respective remaining suffixes of $w$, and therefore after reading $r_{i}$ there are $i$ accepting runs of the previous look-aheads. Suppose that those accepting runs are in the states $Q_{i}$ after reading $r_{i}$. By suitable choices of $L$-components ( $T^{\prime}$ is non-deterministic on $L$-components), we can ensure that there is an accepting run of $T^{\prime}$ such that after reading $r_{i}$ the $L$-component of the states is $Q_{i}$, for all $i$. Conversely, if there is an accepting run of $T^{\prime}$ on $w$, then one can easily reconstruct accepting runs of the look-aheads.

Proof (Proof of Proposition 1). We show that the exponential blow-up already holds with finite state automaton with regular look-ahead. This same blow-up is then obtained for $\mathrm{VPT}_{\text {la }}$ accepting only flat words, i.e. words in $\left(\Sigma_{c} \Sigma_{r}\right)^{*}$ (in that case the stack is useless). On such words each look-ahead visits the suffix of the whole word starting at the position it is triggered. More precisely, we can construct a VPT la such that any VPT needs an exponential number of states to have the same domain of the given VPT. We now show how the exponential blow-up is obtained on finite state automata with look-ahead.

Let $n \in \mathbb{N}$ and $L_{n}=\{v u v|| v \mid=n\} . L_{n}$ is definable by a finite state automaton with look-ahead $A_{n}$ with $O(n)$ states. Let $w=a_{1} \ldots a_{n} w^{\prime}$ and $m=|w|$. For all $1 \leq i \leq n$, when reading $a_{i}, A$ uses a look-ahead to check that the $(m-n+i)$-th letter of $w$ is equal to $a_{i}$. The look-ahead starts in a state $q_{a_{i}, n-i}$, non-deterministically guesses that the current position is the $m-n+i$-th letter of $u$, verifies that it is equal to $a_{i}$ and decreases a counter from $n-i$ to 0 (the counter is implemented with $n-i$ states) to check that the guess was correct. $A_{n}$ as $O(n)$ states.

Without a regular look-ahead, an automaton has to store the $n$-th first letters of $w$ in its states, then it guesses the $m-n$-th position and checks that the prefix of size $n$ is equal to the suffix of size $n$. A simple pumping argument shows that the automaton needs at least $|\Sigma|^{n}$ states. 


\section{B Closure by Visibly Pushdown Look-Ahead Inspecting the Whole Suffix}

We consider in this section VPA that can trigger return transitions on empty stack. Such a VPA is a tuple $\left(Q, q_{0}, F, \Gamma, \delta=\delta_{r} \uplus \delta_{c} \uplus \delta_{\perp}\right)$ where $\delta_{\perp} \subseteq Q \times \Sigma_{r} \times Q$ denotes the set of those new transitions.

We denote by $\mathrm{VPT}_{\text {la }}^{+}$the set of visibly pushdown transducers with look-aheads that can inspect the whole suffix. We prove that VPT are closed by such look-aheads. The construction is done by a slight modification of the construction given in the proof of Theorem 1.

The idea is extend the states a the constructed VPT with a new component $L_{\perp} \subseteq$ $Q^{l a}$ that corresponds to states of look-aheads that started at a deeper position than the current position., and such that any position in between the position at which they started and the current position is not above the current position. For instance, let us consider a well-nested word of the form $w c c_{1} w_{1} r_{1} \ldots c_{n} w_{n} r_{n} r w^{\prime}$ where $w_{i}$ is wellnested. After reading $r_{i}, L_{\perp}$ contains current states of look-aheads that started when reading the words $w_{1}, \ldots, w_{n}$. We therefore have two $L$-components: the look-aheads that started when reading $c_{1}, \ldots, c_{n}$ and the new look-ahead component. When reading $c$, we push on the stack this new component. Let us formally define the construction. From a $\mathrm{VPT}_{\text {la }}^{+} T=\left(Q, q_{0}, F, \Gamma, \delta\right)$ with look-ahead $A=\left(Q^{l a}, q_{0}^{l a}, F^{l a}, \Gamma^{l a}, \delta^{l a}\right)$, we construct an equivalent VPT $T^{\prime}=\left(Q^{\prime}, q_{0}^{\prime}, F^{\prime}, \Gamma^{\prime}, \delta^{\prime}\right)$ possibly exponentially bigger as follows:

- $Q^{\prime}=Q \times 2^{Q^{l a} \times Q^{l a}} \times 2^{Q^{l a}} \times 2^{Q^{l a}}$

- $\Gamma^{\prime}=\Gamma \times 2^{Q^{l a} \times Q^{l a}} \times 2^{Q^{l a}} \times 2^{Q^{l a}} \times \Sigma_{c}$;

- $F^{\prime}=\left\{\left(q, R, L, L_{\perp}\right) \in Q^{\prime} \mid q \in F, L \subseteq F^{l a}, L_{\perp} \subseteq F^{l a}\right\}$.

- $q_{0}^{\prime}=\left(q_{0}, I d_{Q^{l a}}, \varnothing, \varnothing\right)$.

The transitions are defined as follows:

First, for all $q, R, L, L_{\perp}, c, \gamma$, we have:

$\left(q, R, L, L_{\perp}\right) \stackrel{c \mid u,\left(\gamma, R, L \cup\left\{p_{0}\right\}, L_{\perp}, c\right)}{\longrightarrow}\left(q^{\prime}, I d_{Q^{l a}}, \varnothing, \varnothing\right) \in \delta_{c}^{\prime}$ whenever $q \stackrel{c \mid u, p_{0}, \gamma}{\longrightarrow} q^{\prime} \in \delta_{c}$

Then, for all $R, L, L_{\perp}, r, \gamma, q^{\prime \prime}, R^{\prime \prime}, L^{\prime \prime}, L_{\perp}^{\prime \prime}, q^{\prime}, R^{\prime}, L^{\prime}, L_{\perp}^{\prime}$ we have:

$$
\left(q^{\prime \prime}, R^{\prime \prime}, L^{\prime \prime}, L_{\perp}^{\prime \prime}\right) \stackrel{r \mid u,\left(\gamma, R, L, L_{\perp}, c\right)}{\longrightarrow}\left(q^{\prime}, R^{\prime}, L^{\prime}, L_{\perp}^{\prime}\right) \in \delta_{r}^{\prime}
$$

if the following conditions hold:

(i) $q^{\prime \prime} \stackrel{r \mid u, \gamma}{\longrightarrow} q^{\prime} \in \delta_{r}$;

(ii) $R^{\prime}=\left\{\left(p, p^{\prime}\right) \mid \exists s \stackrel{c, \gamma}{\longrightarrow} s^{\prime} \in \delta_{c}^{l a} \cdot \exists\left(s^{\prime}, s^{\prime \prime}\right) \in R^{\prime \prime} \cdot(p, s) \in R\right.$ and $\left.s^{\prime \prime} \stackrel{r, \gamma}{\longrightarrow} p^{\prime} \in \delta_{r}^{l a}\right\}$

(iii) for all $p \in L$ (resp. $L_{\perp}$ ), there exist $p^{\prime} \in L^{\prime}$ (resp. $L_{\perp}^{\prime}$ ), $\gamma \in \Gamma, s, s^{\prime} \in Q^{l a}$ such that $\left(s, s^{\prime}\right) \in R^{\prime \prime}, p \stackrel{c, \gamma}{\longrightarrow} s \in \delta_{c}^{l a}, s^{\prime} \stackrel{r, \gamma}{\longrightarrow} p^{\prime} \in \delta_{r}^{l a}$;

(iv) for all $p \in L_{\perp}^{\prime \prime}$, there exist $p^{\prime} \in L_{\perp}^{\prime}$, such that $p \stackrel{r}{\rightarrow} p^{\prime} \in \delta_{\perp}^{l a}$. 


\section{Decisions Problems}

Proof of Theorem 4, Lower Bound Given $n$ deterministic top-down binary tree automata $T_{1}, \ldots, T_{n}$ over an alphabet $\Delta$, one can construct in linear-time $n$ deterministic VPA $A_{1}, \ldots, A_{n}$ that define the same languages as $T_{1}, \ldots, T_{n}$ respectively, modulo the natural encoding of trees as nested words over the structured alphabet $\tilde{\Delta}=\left\{c_{a} \mid a \in\right.$ $\Delta\} \uplus\left\{r_{a} \mid a \in \Delta\right\}$ [9]. The encoding corresponds to a depth-first left-to-right traversal of the tree. For instance, enc $(f(f(a, b), c))=c_{f} c_{f} c_{a} r_{a} c_{b} r_{b} r_{f} c_{c} r_{c} r_{f}$. We now construct a VPT la $T$ over the alphabet $\tilde{\Delta}$ such that $T$ is functional iff $\bigcap_{i} L\left(T_{i}\right)=\varnothing$. The domain of $T$ are words of the form $w_{n, t}=c_{1} r_{1} \ldots c_{n} r_{n} e n c(t)$ for some ranked tree $t$ over $\Delta$. It is easy to define a VPA that accepts such words and whose size is polynomial in $n$ and $\Delta$. The $n$ first call symbols are used to run $n$ look-aheads. When the $i$-th call $c_{i}$ is read, a look-ahead $B_{i}$ checks that enc $(t) \in L\left(A_{i}\right)$ : it first count that $2(n-i+1)$ symbols have been read and go to the initial state of $A_{i}$. The output words of $T$ are produced as follows: when reading $c_{i}$, there are two possible transitions, that both push the same symbol on the stack, launch the same look-ahead, and goes to the same state, but output two different words, let say $c_{a}$ and $r_{a}$ respectively. If the look-ahead is not accepting the suffix, then none of these transitions can be fired and the computation stops. Any run of $T$ on the word $w_{n, t}$ is accepting. Therefore there is an accepting run of $T$ on $w_{n, t}$ iff $e n c(t) \in \bigcap_{i} L\left(A_{i}\right)$, and in that case there are $2^{n}$ accepting runs whose output words are of the form $\alpha_{1} \ldots \alpha_{n}$ respectively where $\alpha_{i} \in\left\{c_{a}, r_{a}\right\}$. Therefore $T$ is not functional iff there is a tree $t$ such that enc $(t) \in \bigcap_{i} L\left(A_{i}\right)$, iff there is a tree $t$ such that $t \in \bigcap_{i} L\left(T_{i}\right)$. Note that the look-aheads are deterministic.

Proof of the lower bound for Theorem 5 Lower bound The lower-bound in the case where both the transducer and the look-ahead are deterministic is obtained similarly as the lower-bounds for functionality, i.e. by reduction of the emptiness of $n$ deterministic top-down tree automata $T_{1}, \ldots, T_{n}$. We construct two deterministic $\mathrm{VPT}_{\text {la }} T_{1}, T_{2}$ with deterministic look-aheads as in the proof of the lower-bound of Theorem 4 , except that the output words are produced differently. On the word $c_{1} r_{1} \ldots c_{n} r_{n}$ enc $(t)$, the transducer $T_{1}$ (resp. $T_{2}$ ) outputs $c_{a}$ (resp. $r_{a}$ ) when reading $c_{i}$ and only if the lookahead is accepting, i.e. $t \in L\left(T_{i}\right)$. Both transducers are deterministic and as we saw, the look-aheads are also deterministic. Now, the image of $T_{1}$ (resp. $T_{2}$ ) is non-empty iff $\bigcap L\left(T_{i}\right) \neq \varnothing$ iff there exists a tree $t$ such that $\left(w_{n, t}, c_{a}^{n}\right) \in \llbracket T_{1} \rrbracket\left(\right.$ resp. $\left(w_{n, t}, r_{a}^{n}\right) \in$ $\left.\llbracket T_{2} \rrbracket\right)$. Therefore $T_{1}$ and $T_{2}$ are equivalent iff $\bigcap L\left(T_{i}\right)=\varnothing$. 